\title{
Nonparametric estimation of aggregated Sobol' indices: application to a depth averaged snow avalanche model
}

\author{
María Belén Heredia \\ Univ. Grenoble Alpes, INRAE, UR ETNA, Grenoble, France \\ Clémentine Prieur \\ Univ. Grenoble Alpes, CNRS, Inria, Grenoble INP, LJK, Grenoble, France \\ Nicolas Eckert \\ Univ. Grenoble Alpes, INRAE, UR ETNA, Grenoble, France
}

\begin{abstract}
Avalanche models are increasingly employed for elaborating landuse maps and designing defense structures, but they rely on poorly known parameters. Careful uncertainty assessment is thus required but difficulty arises from the nature of the outputs of these models, which are commonly both functional and scalar. Hence, so far in the avalanche field, few sensitivity analyses have been performed. In this work, we propose to determine the most influential inputs of an avalanche model by estimating aggregated Sobol' indices. We propose a nonparametric estimation procedure based on the Nadaraya-Watson kernel smoother, which allows to estimate the aggregated Sobol' indices from a given random sample of small to moderate size. Due to the limited size of the sample, the kernel estimation is biased. Therefore, we propose a bootstrap based bias correction before selecting the bandwidth by cross-validation. After different test-cases showing the efficiency of our approach, it is applied to a real avalanche case. Results show that the friction parameters and the snow depth in the release zone are the most influential parameters determining the avalanche characteristics.
\end{abstract}

\section{Introduction}

In mountain environments, snow avalanches are a constant threat for settlements and their inhabitants [McClung and Schaerer, 2006, Ancey, 2006]. 
This creates conflict between development and safety and land-use planning is an efficient way to reduce death tolls Gruber and Margreth, 2001, Eckert et al. 2018. To this aim, avalanche flow dynamic models are increasingly employed for elaborating land-use maps and designing defense structures Naaim et al., 2010, Favier et al., 2014]. However, all existing physicallybased snow avalanche models remain based on some parameters which are poorly known Ancey and Meunier, 2004, Eckert et al., 2008. This applies, for instance, to friction parameters representing the behavior of snow in motion and to initial conditions corresponding to the avalanche release Jamieson et al., 2008, Eckert et al., 2010, Naaim et al., 2013. Thus, a careful assessment of the impact of the uncertainty of the inputs on the outputs should be carried out Fischer, 2013. Difficulty arises from the nature of the outputs of these models, which are commonly both functional (e.g., the velocity and flow depth of the avalanche as function of space and time) and scalar (e.g., the runout distance, aka the point of further reach on the $2 \mathrm{D}$ topography of the avalanche flow path).

One of the main purposes of sensitivity analysis is to determine the inputs which are the most influential on the output or outputs of a model where the model can be of any nature, for example a black-box model or a complex computational code [Saltelli et al., 2000]. More specifically, Saltelli et al. 2004] proposed four settings as a guide of objectives of a sensitivity analysis: factor prioritization, factor fixing, variance cutting and factor mapping Saltelli et al., 2004, Iooss and Prieur, 2017. It depends on the analyst to define the objectives of its sensitivity analysis. In the global sensitivity (GSA) framework, the input parameters are modeled by random variables, which will be assumed independent in this paper. The probability distribution chosen to model the input vector is often guided by practitioner's belief. The output is then random as it depends on the inputs through the model. In this work, our objective is factor prioritization which consists in identifying which inputs or factors once fixed would reduce the variance of the output [Saltelli et al., 2008] at most.

There exist different sensitivity measures in the literature for quantifying the influence of each input on the output: variance based indices, also known as Sobol' indices [Sobol', 1993], density based measures [Borgonovo et al., 2016], entropy-based sensitivity measures [Auder and Iooss, 2008], etc. A detailed review of sensitivity measures can be found, e.g., in Iooss and Lemaître 2015] and Borgonovo and Plischke [2016].

We focus our study on variance based Sobol' indices [Sobol', 1993] commonly used for factor prioritization. Moreover, Sobol' indices have a natural extension to multivariate or functional outputs [see, e.g., Lamboni et al., 
2009, Gamboa et al., 2013 which is the framework of the application that motivated this study. Another remarkable advantage of Sobol' indices is their easy interpretation: a high index shows the input is relevant and a value close to zero shows that it is not, all the indices are normalized between 0 and 1 and sum to 1 .

In short, Sobol' indices are constructed as follows: if the output of a model is scalar, its total variance can be split into partial variances by using the Hoeffding decomposition Hoeffding, 1948]. If the inputs are independent, each of the partial variances is associated to an input or to an interaction between inputs through the ANOVA decomposition Efron and Stein, 1981]. Then, the Sobol' indices [Sobol', 1993 are calculated as the ratio of each partial variance and the total variance.

If the output is multivariate, it is possible to apply sensitivity analysis to each component of the multivariate output but this could lead to redundancies in the results, particularly in the setting of discretized functional outputs. It seems therefore interesting to turn to the so-called aggregated indices first introduced in Lamboni et al. [2009] which summarize the information. A preliminary step of output dimension reduction such as, e.g., principal component analysis (PCA) or partial least-squares may also be applied first [see, e.g., Campbell et al., 2006]. For our real application in the avalanche field, dimension reduction was performed by simultaneous PCA introduced in Ramsay and Silverman 2005] [see also Nanty et al., 2017, and references therein].

We now discuss the estimation of aggregated Sobol' indices. There are many methods to estimate Sobol' indices: the Fast Amplitude Sensitivity Test (FAST) [see, e.g., Saltelli et al., 1999, and references therein], Random Balance Design Tarantola et al., 2006], Sobol' pick-freeze schemes [Sobol', 1993. The main drawback of all the aforementioned methods Plischke, 2010 is that they are based on sampling designs of particular type. Nevertheless, for many memory and time consuming real applications, the cost (in terms of number of model evaluations) of these approaches is prohibitive. For example, to estimate a single Sobol' index with an uncertainty of $10 \%$, it could be required to perform $10^{4}$ model runs Iooss and Lemaitre, 2015.

To overcome the drawback of the aforementioned methods, authors use approaches not based on structured sampling designs to estimate the indices. These methods are known as given data or one sample methods. They also correspond to green sensitivity analysis because available data from previous model runs can be reused. Among these approaches, let us cite the effective algorithm for computing global sensitivity indices (EASI) method proposed by Plischke [2010], which is a spectral method based on the Fast 
Fourier Transform. We also mention the work in Plischke et al. [2013], which relies on the notion of class-conditional densities, where a class is a sub-sample stemming from a suitable partition of the dataset. Much more recently, Antoniano-Villalobos et al. 2019 proposed a fully Bayesian given data procedure.

In the present paper, we propose a new given data method to estimate first-order Sobol' indices, based on a nonparametric Nadaraya-Watson Nadaraya, 1964, Watson, 1964 bias corrected kernel regression Racine, 2001]. Our approach is close to the one introduced in Solís [2019]. The main difference is in the formula we apply to correct the bias, which seems to be more efficient, at least in the results of our simulation study. We then extend this estimation procedure to the estimation of the aggregated Sobol' indices [Lamboni et al., 2009]. To our knowledge, it is the first time a nonparametric given data procedure is proposed in this framework. In the framework of the estimation of non aggregated usual Sobol' indices, Da Veiga et al. [2009] proposed a methodology based on local linear regression. The Nadaraya-Watson estimator can be seen as a particular case of the wider class of nonparametric estimators, called local polynomial estimators used in Da Veiga et al. 2009]. Specifically, it corresponds to performing a local constant fit. However the procedure in Da Veiga et al. 2009] is clearly different from ours, as it consists in spliting the sample in two disjoint sub-samples, the first one used to emulate the conditional expectation, the second one used to estimate each first-order Sobol' index, replacing the conditional expectation with its corresponding emulator. More recently, Broto et al. 2018] proposed to estimate Shapley effects introduced by Owen [2014 in the framework of sensitivity analysis with a nonparametric estimator based on nearest neighbours.

Within the avalanche field, only a few studies have analyzed the influence of inputs on the outputs of dynamic propagation models. For example, Barbolini and Savi 2001] used a Monte Carlo approach to analyze the sensitivity of runout distances and impact pressures in the VARA model. Jamieson et al. 2008 discussed the main sources of uncertainty in the inputs of avalanche models. Borstad and McClung 2009 developed a sensitivity analysis of an avalanche model with a Coulomb-type friction law. Bühler et al. 2011 explored the influence of the digital elevation models resolution on the outputs of the RAMMS avalanche model. Eventually, Bühler et al. 2018 developed a sensitivity analysis of released volumes, runout distances and avalanche velocities in the runout zone with respect to the initial released volume with the RAMMS avalanche model. However, the previous studies did not apply formal statistical methods to quantify the respective 
importance of the inputs. Moreover, authors considered only scalar outputs in their approaches. By contrast, in our paper, we focus on the avalanche model proposed by Naaim et al. 2004] and we quantify the importance of its inputs on its outputs by estimating the aggregated Sobol' indices with our new nonparametric procedure. The uncertainty on the input parameters was defined based on data obtained from an avalanche released on 13 February 2013 at the Lautaret full-scale test-site [Thibert et al., 2015].

In summary, the aims of this study are: (i) to propose a nonparametric estimation method for the aggregated Sobol' indices in a given data framework when sample size is small (ii) to quantify the input importance in avalanche models, having complex outputs (e.g., a mix of functional and scalar outputs). The approach can be easily adapted to other avalanche models and more widely in environmental sciences in the frequent case of complex models with outputs which are both functional and scalar. This work is organized as follows: the aggregated Sobol' indices and the estimation method are described in Section 2. In Section 3, we test the estimation method on toy functions. Then, in Section 4 , the avalanche model is described and the results are presented. Finally, in Section 5, the conclusions and perspectives are discussed.

\section{Aggregated Sobol' indices}

Let us denote by $f$ the model which takes as inputs the vector $\mathbf{X}=\left(X_{1}, \ldots, X_{d}\right)$. The inputs $X_{1}, \ldots, X_{d}$ are modeled by random variables defined on a probability space $(\Omega, \mathcal{F}, \mathbb{P})$ and valued in a measurable space $E=E_{1} \times E_{2} \ldots \times E_{d}$. The output of the model $f$ is the $p$ multivariate vector $\mathbf{Y}=\left(Y_{1}, \ldots, Y_{p}\right)^{T}$. It means, we have:

$$
f(\mathbf{X})=f\left(X_{1}, \ldots, X_{d}\right)=\mathbf{Y}=\left(Y_{1}, \ldots, Y_{p}\right)^{T} .
$$

In the following, we assume that $\mathbb{E}\left(Y_{1}^{2}+\ldots+Y_{p}^{2}\right)<\infty$ and that the random variables $X_{1}, \ldots, X_{d}$ are independent from each other. This condition guarantees the unicity of the ANOVA decomposition [Sobol', 1993]. To summarize the importance of each input $X_{i}$ on the multivariate output $\mathbf{Y}$, we aim at computing the aggregated Sobol' index $G S_{i}$ introduced in Lamboni et al. 2009] [see also Gamboa et al., 2013] defined as:

$$
G S_{i}=\frac{\sum_{j=1}^{p} \operatorname{Var}\left(Y_{j}\right) S_{i}^{j}}{\sum_{j=1}^{p} \operatorname{Var}\left(Y_{j}\right)}
$$


where $S_{i}^{j}$ is the first-order Sobol' index of the output $Y_{j}$ with respect to the input $X_{i}$ namely

$$
S_{i}^{j}=\frac{\operatorname{Var}\left(\mathbb{E}\left(Y_{j} \mid X_{i}\right)\right)}{\operatorname{Var}\left(Y_{j}\right)}
$$

Notice that $G S_{i} \in[0,1]$. As in the case of the scalar indices, the main advantage of the aggregated indices is their easy interpretation: a high value means the input is important, a value close to zero means it is not, and $\sum_{i=1}^{d} G S_{i}=1$.

In the following, we propose to estimate nonparametrically $G S_{i}$ from given data, proposing a new procedure based on the Nadaraya-Watson kernel smoother Nadaraya, 1964, Watson, 1964.

\subsection{Nonparametric estimation procedure}

Let $\left(X_{i}^{\ell}, Y_{j}^{\ell}\right)_{1 \leq \ell \leq n}$ be an independent identically distributed random sample of $\left(X_{i}, Y_{j}\right)$. The Nadaraya-Watson kernel smoother consists in estimating the conditional mean of $\mathbb{E}\left(Y_{j} \mid X_{i}\right)$ at a point $x$ in the domain of $X_{i}$ by:

$$
\mathbb{E}\left(Y_{j} \mid X_{i}=x\right)=m_{h}(x)=\sum_{\ell=1}^{n} Y_{j}^{\ell} W_{\ell, h}(x),
$$

where

$$
W_{\ell, h}(x)=\frac{K\left(\frac{x-X_{i}^{\ell}}{h}\right)}{\sum_{\ell=1}^{n} K\left(\frac{x-X_{i}^{\ell}}{h}\right)},
$$

with $K$ a kernel function and $h$ a bandwidth. In Equation (3), different weights $W_{\ell, h}$ can be used, as for example, local linear regression proposed in Da Veiga et al. 2009 and nearest neighbor smoothers as proposed by Broto et al. [2018].

When the sample size is small, the selection of the bandwidth $h$ is critical. Indeed, to avoid undersmoothing or oversmoothing, an optimal value of $h$ which balances bias and variance must be found [Tsybakov, 2008, page 17]. An option widely used to select $h$ is cross-validation but if the sample size is small, the estimator will suffer of finite-sample bias [see Racine, 2001, Solís, 2019. Therefore, if the sample size is small, it is recommended to perform a bias correction to the kernel smoother estimator preliminary to the bandwidth selection. 
It is important to underline that the criterion we introduce here to select the badwidth is different from the one proposed in Solís [2019], which was based on bagging minimization. The selection of the bandwidth we propose to apply in our paper is described in the next section.

Once the bandwidth $h$ has been chosen, the estimation of the aggregated Sobol' index is straightforward: given a $n$ sample $\left(x_{i}^{\ell}, y_{j}^{\ell}\right)_{1 \leq \ell \leq n}$ of $\left(X_{i}, Y_{j}\right)$,

$$
\widehat{G S}_{i}=\frac{\sum_{j=1}^{p} \widehat{\operatorname{Var}}\left[\left(y_{j}^{\ell}\right)_{1 \leq \ell \leq n}\right] \hat{S}_{i}^{j}}{\sum_{j=1}^{p} \widehat{\operatorname{Var}}\left[\left(y_{j}^{\ell}\right)_{1 \leq \ell \leq n}\right]}
$$

where $\hat{S}_{i}^{j}$ is the estimation of the first-order Sobol' index:

$$
\hat{S}_{i}^{j}=\frac{\widehat{\operatorname{Var}}\left[\left(\widehat{m}_{\mathrm{h}}\left(x_{i}^{\ell}\right)\right)_{1 \leq \ell \leq n}\right]}{\widehat{\operatorname{Var}}\left[\left(y_{j}^{\ell}\right)_{1 \leq \ell \leq n}\right]},
$$

with $\widehat{\operatorname{Var}}$ denoting empirical variance.

Note that the same sample $\left(x_{i}^{\ell}, y_{j}^{\ell}\right)_{1 \leq \ell \leq n}$ is used for both the estimators $\hat{m}_{h}$ and $\widehat{\operatorname{Var}}$.

\subsection{Bandwidth selection $h$ with bias correction}

As mentioned previously, cross-validation is one option to select the bandwidth. In more details, given a $n$ sample $\mathbf{x}=\left(x^{1}, \ldots, x^{n}\right)$ of $X_{i}$ and the corresponding evaluations $\mathbf{y}=\left(y^{1}, \ldots, y^{n}\right)$ of $Y_{j}$, the bandwidth can be selected as:

$$
h_{\mathrm{cv}}=\operatorname{argmin}_{h>0} \frac{1}{n} \sum_{k=1}^{n}\left(y^{k}-\frac{\sum_{\substack{\ell=1 \\ \ell \neq k}}^{n} y^{\ell} K_{h}\left(x^{\ell}-x^{k}\right)}{\sum_{\substack{\ell=1 \\ \ell \neq k}}^{n} K_{h}\left(x^{\ell}-x^{k}\right)}\right)^{2},
$$

for notation simplicity, $K_{h}($.$) denotes K(\dot{\bar{h}})$ and we dropped all the $i$ and $j$ subindices, but keep in mind that we refer to a sample of $X_{i}$ and $Y_{j}$.

If the sample size is small, it is better to apply a bias correction before performing bandwidth selection. In our framework of sensitivity analysis, we adapt the procedure introduced in Racine 2001 to select the bandwidth. The different steps of our procedure are described hereafter:

1. Given a $n$ sample $\mathbf{x}=\left(x^{1}, \ldots, x^{n}\right)$ of $X_{i}$ and the corresponding evaluations $\mathbf{y}_{j}=\left(y^{1}, \ldots, y^{n}\right)$ of $Y_{j}$ and a bandwidth $h>0$ (for example, 
an initial bandwidth value could be $h=h_{\mathrm{cv}}$ given by Equation (5)), we calculate the Nadaraya-Watson kernel regression estimate:

$$
\widehat{m}_{h}\left(x^{k}\right)=\frac{\sum_{\substack{\ell=1 \\ \ell \neq k}}^{n} y^{\ell} K_{h}\left(x^{\ell}-x^{k}\right)}{\sum_{\substack{\ell=1 \\ \ell \neq k}}^{n} K_{h}\left(x^{\ell}-x^{k}\right)} .
$$

If $\sum_{\substack{\ell=1 \\ \ell \neq k}}^{n} K\left(x^{\ell}-x^{k}\right)=0$, we set $\widehat{m}_{h}\left(x^{k}\right)=0$.

2. We calculate the errors:

$$
\hat{\epsilon}^{\ell}=y^{\ell}-\widehat{m}_{h}\left(x^{\ell}\right), \quad \ell=1, \ldots, n .
$$

These errors are heteroscedastic because $Y_{j}$ depends also on $X_{1}, \ldots, X_{i-1}, X_{i+1}, \ldots, X_{d}$, therefore, a standardization of the errors must be applied before bootstraping.

3. The errors $\left\{\hat{\epsilon}^{\ell}\right\}_{1 \leq \ell \leq n}$ are standardized as:

$$
\hat{v}^{\ell}=\frac{\hat{\epsilon}^{\ell}-\bar{\epsilon}}{r\left(x^{\ell}\right)}, \quad \ell=1, \ldots, n
$$

where $\bar{\epsilon}$ is the empirical mean of $\left\{\hat{\epsilon}^{\ell}\right\}_{1 \leq \ell \leq n}$ and $r\left(x^{\ell}\right)$ is an estimation of the conditional standard deviation of the random variable $\epsilon \mid X_{i}$ at $x . r\left(x^{\ell}\right)$ is estimated using kernel smoother nonparametric estimation.

4. A bootstrap sample $\mathbf{y}^{(b)}$ of $\mathbf{y}$ is created as:

$$
y^{(b) \ell}=\widehat{m}_{h}\left(x^{\ell}\right)+r\left(x^{\ell}\right) v^{(b) \ell}, \quad \ell=1, \ldots, n,
$$

where $v^{(b) \ell}$ is a bootstrap sample of $\left\{\hat{v}^{\ell}\right\}_{1 \leq \ell \leq n}$.

5. $B$ bootstrap samples of $\mathbf{y}$ are created using Step 4. For each bootstrap sample $\left(\mathbf{x}, \mathbf{y}^{(b)}\right)$, we estimate the kernel smoother $\hat{m}_{h}^{(b)}$ using Equation (6).

6. The bias of $\hat{m}_{h}\left(x^{\ell}\right)$ is estimated as:

$$
\frac{1}{B} \sum_{b=1}^{B} \widehat{m}_{h}^{(b)}\left(x^{\ell}\right)-\hat{m}_{h}\left(x^{\ell}\right)
$$


Then, the corrected bias kernel smoother $\hat{m}_{h}^{c}\left(x^{\ell}\right)$ is calculated as:

$$
\begin{aligned}
\hat{m}_{h}^{c}\left(x^{\ell}\right) & =\hat{m}_{h}\left(x^{\ell}\right)-\left(\frac{1}{B} \sum_{b=1}^{B} \widehat{m}_{h}^{(b)}\left(x^{\ell}\right)-\hat{m}_{h}\left(x^{\ell}\right)\right) \\
& =2 \hat{m}_{h}\left(x^{\ell}\right)-\frac{1}{B} \sum_{b=1}^{B} \widehat{m}_{h}^{(b)}\left(x^{\ell}\right)
\end{aligned}
$$

7. Finally, the bandwidth $h$ is selected as

$$
\hat{h}_{\text {boot }}=\operatorname{argmin}_{h>0} \frac{1}{n} \sum_{\ell=1}^{n}\left(y^{\ell}-\hat{m}_{h}^{c}\left(x^{\ell}\right)\right)^{2} .
$$

Once $\hat{m}_{\text {boot }}^{c}$ is calculated, the aggregated Sobol' index $G S_{i}$ is estimated from (4). In what follows, our estimator with bias correction is denoted by cnp and the same estimator without bias correction, for which the bandwidth is selected by solving (5), is denoted by np.

\subsection{Dimension reduction based on principal component anal- ysis}

If the output is high-dimensional or even functional, it may be computationally interesting to reduce the output dimension in a preliminary step. There are different reduction techniques. Principal component analysis, also known as Karhunen-Loève decomposition in the functional framework, consists in projecting the output on a new basis so that most information is concentrated in the first few components [see, e.g., Pearson, 1901, Loève, 1963]. Note that if the outputs are the descretization of more than one functional random variable (e.g., velocity and flow depth), simultaneous PCA may provide efficient reduction. For more details, see e.g., the work of Nanty et al. 2017 and reference therein.

In brief, simultaneous PCA consists in applying PCA to the matrix composed by the concatenation of the discretized functions divided by a normalization factor. Nanty et al. 2017] show that using the maximum of each functional as normalization factor provides the best results. Thus, in the avalanche application, we follow their procedure.

There exist dimension reduction tools specific to functional data, such as the regularized functional PCA described in Chapter 9 of Ramsay and Silverman 2005] [see also the work of Yao et al., 2005] which has been 
applied in different fields [e.g., Locantore et al., 1999, Antoniadis et al., 2012. Also, it has been shown that in some applications, functional PCA provides better results than usual PCA applied after a discretization of the functional data [e.g., Viviani et al., 2005]. On our test cases, usual PCA applied on the discretized data set and functional PCA provided similar results. The results we show in this paper are the ones obtained by applying usual PCA on discretized data.

The PCA consists in decomposing the variance-covariance matrix of the output. More precisely, let us denote by $\Sigma$ the variance-covariance matrix of the output vector $\mathbf{Y}$. The principal component decomposition of $\mathbf{Y}$ is based on the expansion:

$$
\Sigma=\sum_{j=1}^{p} \lambda_{j} \mathbf{v}_{j} \mathbf{v}_{j}^{T},
$$

where $\lambda_{1} \geq \ldots \geq \lambda_{p}$ are the eigenvalues and $\mathbf{v}_{1}, \ldots \mathbf{v}_{p}$ are the orthonormal eigenvectors of $\Sigma$. Therefore, $\mathbf{Y}$ can be decomposed as:

$$
\begin{aligned}
\mathbf{Y} & =\mathbb{E}(\mathbf{Y})+\sum_{j=1}^{p}\left((\mathbf{Y}-\mathbb{E}(\mathbf{Y}))^{T} \mathbf{v}_{j}\right) \mathbf{v}_{j} \\
& =\mathbb{E}(\mathbf{Y})+\sum_{j=1}^{p} \mathbf{h}_{j} \mathbf{v}_{j}
\end{aligned}
$$

where $\mathbf{h}_{j}$ is the $j t h$ principal component of $\mathbf{Y}$. The output can be approximated using the $q \leq p$ first components which capture the major part of the output variance:

$$
\mathbf{Y} \approx \mathbb{E}(\mathbf{Y})+\sum_{j=1}^{q} \mathbf{h}_{j} \mathbf{v}_{j} .
$$

Then, the aggregated Sobol' indices can be computed in the first $q$ principal components. For example, for input $X_{i}$ :

$$
G S_{i} \approx \frac{\sum_{j=1}^{q} \lambda_{j} S_{i}\left(\mathbf{h}_{j}\right)}{\sum_{j=1}^{q} \lambda_{j}}
$$

where $S_{i}\left(\mathbf{h}_{j}\right)$ denotes the Sobol' index of $\mathbf{h}_{j}$ with respect to input $X_{i}$.

The estimation of the aggregated Sobol' indices will be done for the reduced output $\mathbf{H}=\left(h_{1}, \ldots, h_{q}\right)^{T}$. 


\section{Test cases}

The cnp method is tested on three toy functions and then, it is applied to an avalanche dynamic model. The cnp accuracy is compared with $\mathrm{np}$ (bandwidth selected with cross-validation and without bias correction), Solis2019 Solís, 2019], and sobolroalhs [Tissot and Prieur, 2015 implemented in R. The estimator implemented in sobolroalhs is a pick-and-freeze method, which is particularly interesting in terms of number of model evaluations required for an accurate estimation of Sobol' indices.

Because of its regularity properties, the Epanechnikov kernel of order 2 is used:

$$
K(u)=\frac{3}{4}\left(1-u^{2}\right) I_{(|u| \leq 1)},
$$

where $I$ denotes the indicator function. All the computations were performed in R Core Team 2019.

\subsection{Toy functions}

For each toy function, to test the accuracy of the method, the Sobol' indices were estimated with $N=100$ independent samples of sizes $n \in$ $\{100,200,300\}$. For cnp, the number of bootstrap samples was set to $B=$ 100 .

\subsubsection{Scalar g-sobol function}

The scalar g-Sobol function is defined as:

$$
Y=f\left(X_{1}, \ldots, X_{d}\right)=\prod_{i=1}^{d} \frac{\left|4 X_{i}-2\right|+a_{i}}{1+a_{i}}, \quad a_{i} \in \mathbb{R}, i \in\{1, \ldots d\}
$$

where $\left(X_{1}, \ldots, X_{d}\right) \sim \mathcal{U}\left([0,1]^{d}\right)$.

An analytical expression of the scalar Sobol' indices is available for this test case:

$$
S_{i}=\frac{\frac{1}{3\left(1+a_{i}\right)^{2}}}{\prod_{i=1}^{d}\left(\frac{3\left(a_{i}\right)^{2}+6 a_{i}+4}{3\left(1+a_{i}\right)^{2}}\right)-1}, \quad i \in\{1, \ldots, d\} .
$$

For this first test case, we chose $d=8$ and $\mathbf{a}=(0,1,4.5,9,99,99,99,99)$. The indices were estimated for $N=100$ independent samples of sizes 
$n \in\{100,200,300\}$ using the four methods cnp, np, sobolroalhs and Solis2019. The results can be seen in Figures 1 and 2 .

We remark that the cnp procedure we have proposed in this work behaves well even for small data sets. The bias correction seems to outperform the usual np procedure, except maybe for $n=100$.

The variances of both $\mathrm{cnp}$ and $\mathrm{np}$ are improved with respect to pickfreeze procedure sobolroalhs based on replicated sampling of [Tissot and Prieur, 2015 and even to the nonparametric bagging approach in Solís, 2019]. At least, we remark a decrease of the efficiency of our approach for very small Sobol' indices.

\subsubsection{Multivariate g-sobol function}

To test our estimation method on a multivariate function, we use a multivariate version of the $\mathrm{g}$-Sobol function. The function is defined as:

$$
\left(Y_{1}, \ldots, Y_{p}\right)^{T}=f\left(X_{1}, \ldots, X_{d}\right) \text { with }\left(X_{1}, \ldots, X_{d}\right) \sim \mathcal{U}\left([0,1]^{d}\right)
$$

where

$$
Y_{j}=\prod_{i=1}^{d} \frac{\left|4 X_{i}-2\right|+a_{i}^{j}}{1+a_{i}^{j}}, a_{i}^{j}, \text { with } j \in\{1, \ldots, p\}, i \in\{1, \ldots d\} .
$$

An analytical expression of aggregated Sobol' indices is available for this test case:

$$
G S_{i}=\frac{\sum_{j=1}^{p} \frac{1}{3\left(1+a_{i}^{j}\right)^{2}}}{\sum_{j=1}^{p} \prod_{i=1}^{d}\left(\frac{3\left(a_{i}^{j}\right)^{2}+6 a_{i}^{j}+4}{3\left(1+a_{i}^{j}\right)^{2}}\right)-1} .
$$

We chose $p=2, d=6$ and the coefficients $a_{i}^{j}, i \in\{1, \ldots, 6\}, j \in\{1,2\}$ are coded in the matrix $A_{2} \in \mathbb{R}^{6 \times 2}$ :

$$
A_{2}^{T}=\left(\begin{array}{cccccc}
0 & 0.5 & 3 & 9 & 99 & 99 \\
1 & 1 & 1 & 1 & 1 & 1
\end{array}\right)
$$

Figure 3 shows the estimation of the Sobol' indices using cnp, np and sobolroalhs in $N=100$ independent samples of sizes $n \in\{100,200,300\}$. The bias accuracy of cnp compared to the other two methods is the best (see Figure 3). Furthermore, the variance of cnp estimation is lower than 

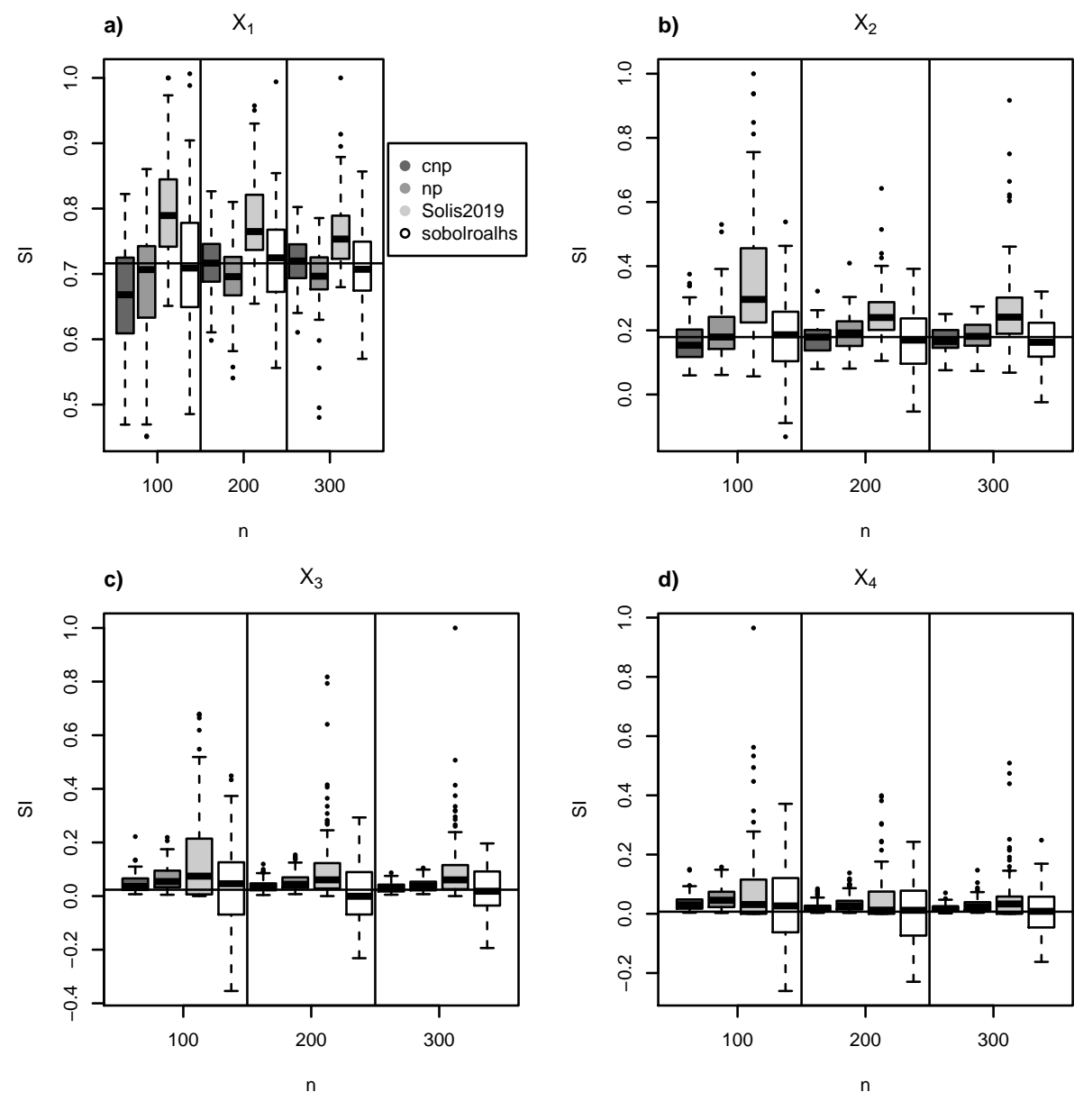

Figure 1: Scalar g-Sobol function: estimation of the first-order Sobol' indices for $X_{1}, X_{2}, X_{3}$ and $X_{4}$ using four estimation methods: cnp, np, Solis2019 and sobolroalhs. To draw the boxplots, the indices were computed in $N=100$ independent samples of sizes $n \in\{100,200,300\}$. For each iteration of cnp and Solis2019, we used $B=100$ bootstrap samples. 

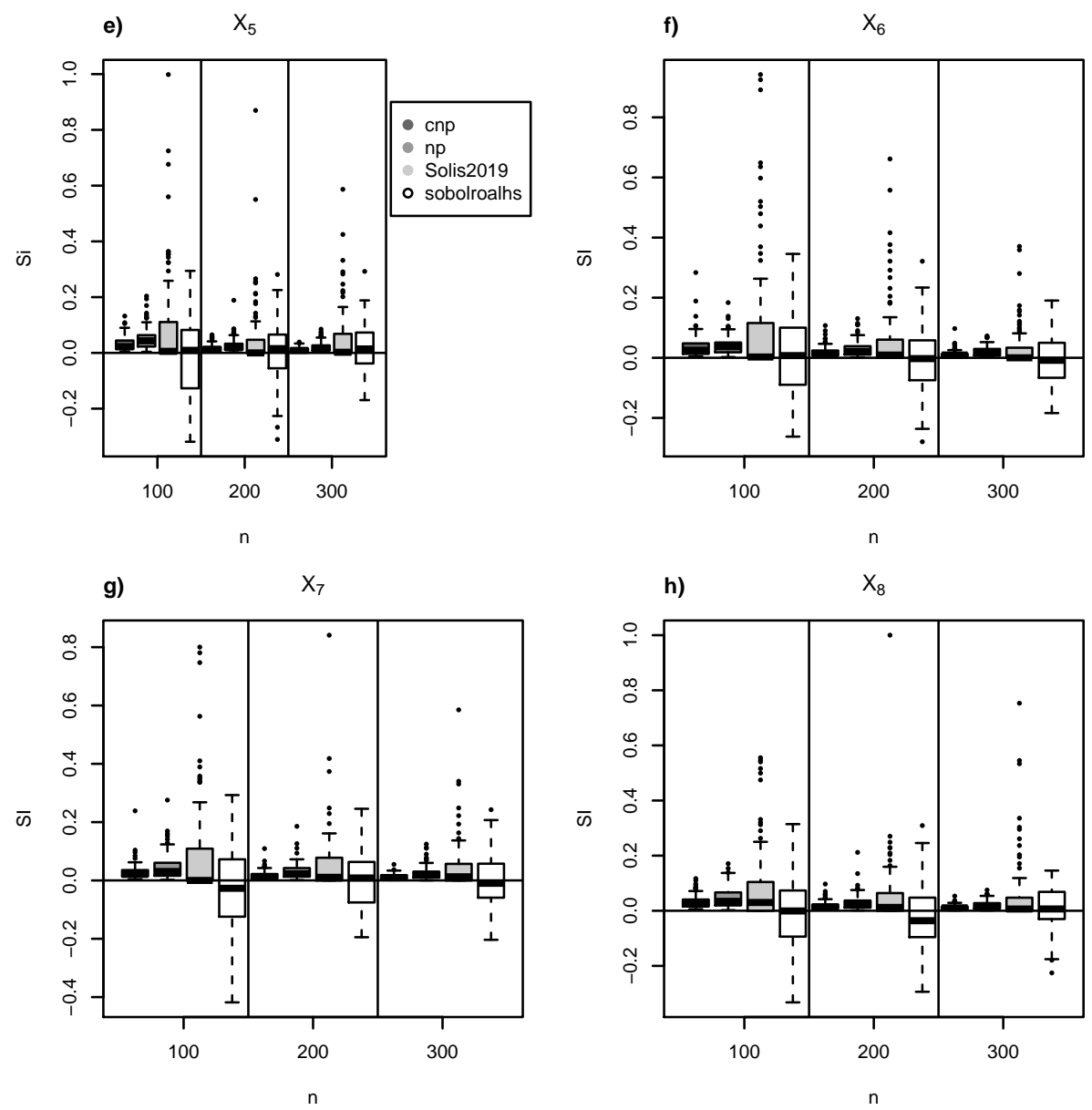

Figure 2: Scalar g-Sobol function: estimation of the first-order Sobol' indices for $X_{5}, X_{6}, X_{7}$ and $X_{8}$ using four estimation methods: cnp, np, Solis2019 and sobolroalhs. To draw the boxplots, the indices were computed in $N=100$ independent samples of sizes $n \in\{100,200,300\}$. For each iteration of cnp and Solis2019, we used $B=100$ bootstrap samples. 


\begin{tabular}{|c|l|l|}
\hline Input & Description & Distribution \\
\hline$m$ & mass $(\mathrm{kg})$ & $\mathcal{U}[10,12]$ \\
$c$ & damping constant $\left(\mathrm{Nm}^{-1} \mathrm{~s}\right)$ & $\mathcal{U}[0.4,0.8]$ \\
$k$ & spring constant $\left(\mathrm{Nm}^{-1}\right)$ & $\mathcal{U}[70,90]$ \\
$l$ & initial elongation $(\mathrm{m})$ & $\mathcal{U}[-1,-0.25]$ \\
\hline
\end{tabular}

Table 1: Mass spring model: Inputs description and uncertainty intervals.

the other two methods (see Figure 3). Additionally, for this test case, the bias correction performance is more evident than in the previous case (see Figures 1 and 2 compared to Figure 3 ).

\subsection{A functional example: the mass-spring model}

Before applying our procedure to the avalanche application, we illustrate its performance on a test case with discretized functional output: the functional mass-spring model proposed by Gamboa et al. [2013, where the displacement of a mass connected to a spring is considered:

$$
m x^{\prime \prime}(t)+c x^{\prime}(t)+k x(t)=0,
$$

with initial conditions $x(0)=l, x^{\prime}(0)=0$, and $t \in[1,40]$. There exists an analytical solution of Equation (10). This model has four inputs (see more details in Table 1). The model output is the vector:

$$
\mathbf{Y}=f(\mathbf{X})=\left(x\left(t_{1}\right), \ldots, x\left(t_{800}\right)\right), \quad t_{i}=0.05 i \text { with } i \in\{1, \ldots, 800\} .
$$

The discretized output is high-dimensional $(p=800)$. Therefore, we first reduce the dimension of the output by using PCA before estimating the aggregated Sobol' indices in the first $q$ principal components with $q \ll p$.

The indices were estimated for $N=100$ independent samples of size $n \in\{100,200,300\}$. Each sample was also used for PCA. We tested if the results were more accurate if we used two independent samples, one for PCA and the other one for the estimation of the Sobol' indices. At a constant cost in terms of model evaluations, the results were even better using only one sample (see Figure 12 in the Appendix in Section 6).

The explained variance as a function of the number of PCs $q$ is shown in Figure 4. Notice that $70 \%$ of the variance is explained with a small number of PCs $(q=3)$ and almost all the variance $(95 \%)$ is explained by 6 PCs. Thus, the aggregated Sobol' indices are estimated using the first 6 PCs. 

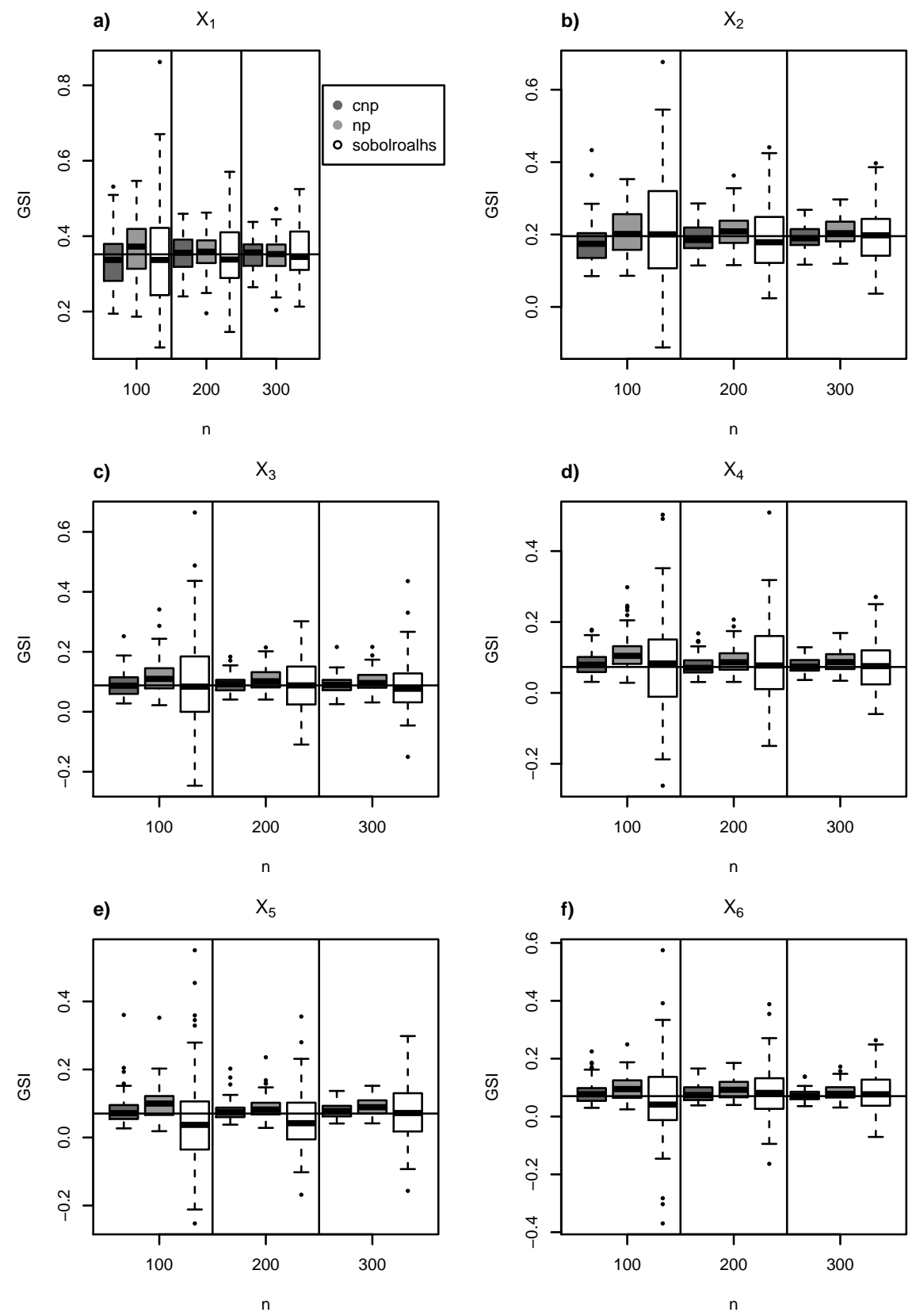

Figure 3: Multivariate g-Sobol function: estimation of the aggregated Sobol' indices using cnp, np and sobolroalhs. Boxplots were drawn from $\mathrm{N}=100$ samples of sizes $n \in\{100,200,300\}$. To use cnp, we set $B=100$. 


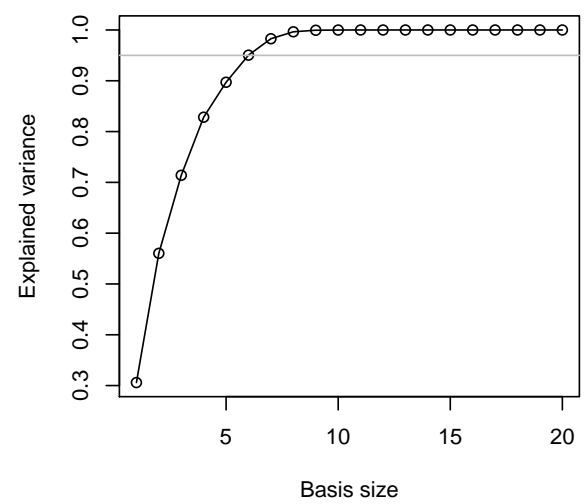

Figure 4: Mass-spring model: Explained variance as a function of the decomposition basis size (number of PCs). The gray line is displayed at the $95 \%$ of the variance explained which corresponds to 6 PCs.

The true aggregated Sobol' indices for this instance are unknown but they are considered close to the values estimated with a sample of large size $n=100000$ using sobolroalhs.

The evolution of the estimation of the aggregated Sobol' indices using the three methods in function of the sample size $n \in\{100,200,300\}$ are shown in Figure 5. The cnp estimation has a much smaller variance than the sobolroalhs estimation. The bias correction allows to correct the bias of the $\mathrm{np}$ estimation procedure, even if a residual bias seems to persist. The bias decreases as $n$ increases, as expected. As it was mentioned before, our aim is factor prioritization and this objective is achieved with the cnp method in the sense that the most influential factor $X_{3}$ is clearly identified.

In general, the cnp method has a better accuracy compared to the other methods np, sobolroalhs and Solis2019 in all the test cases we considered. Therefore, in the following section we apply the cnp method to estimate the aggregated Sobol' indices of an avalanche model.

\section{Application: the avalanche model}

The avalanche model used in this study represents the avalanche motion as a fluid. The model is based on depth-averaged Saint-Venant equations and it was proposed by Naaim et al. 2004. 

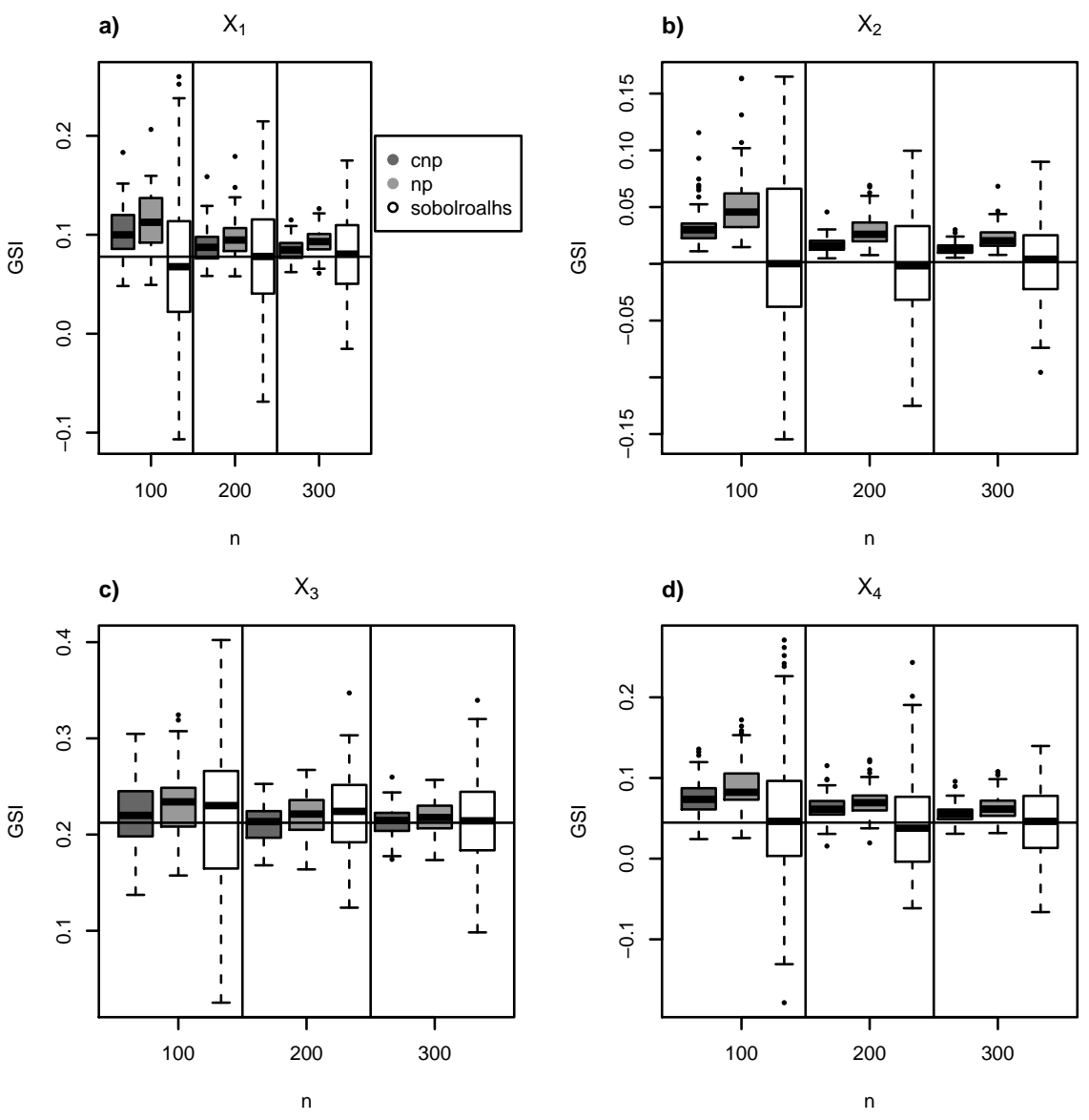

Figure 5: Mass-spring model: Estimation of the aggregated Sobol' indices using cnp, $\mathrm{np}$ and sobolroalhs. Boxplots were drawn from $\mathrm{N}=100$ samples of different sizes $n \in\{100,200,300\}$. To use cnp, we set $B=100$. 
In short, the model considers only the dense layer of the avalanche. The depth of the flow is then small compared to its length. Under this assumption, shallow-water approximations of the mass and momentum equations can be used. Also, the model assumes the avalanche is flowing on a curvilinear profile $z=l(x)$, where $z$ is the elevation and $x$ is the projected runout length distance measured from the starting abscissa point of the avalanche. The mass and momentum conservation equations of the SaintVenant avalanche model are:

$$
\begin{aligned}
\frac{\partial h}{\partial t}+\frac{\partial h v}{\partial x} & =0 \\
\frac{\partial h v}{\partial t}+\frac{\partial}{\partial x}\left(h v^{2}+\frac{h^{2}}{2}\right) & =h(g \sin \phi-\mathrm{F})
\end{aligned}
$$

where $v=\|\overrightarrow{\mathbf{v}}\|$ is the flow velocity, $h$ is the flow depth, $\phi$ is the local angle, $t$ is the time, $g$ is the gravity constant and $\mathrm{F}=\|\overrightarrow{\mathbf{F}}\|$ is a frictional force. The $\mathrm{F}$ frictional force considered in the model is the Voellmy frictional force:

$$
\mathrm{F}=\mu g \cos \phi+\frac{g}{\xi h} v^{2}
$$

where $\mu$ and $\xi$ are the friction parameters (see more details of the avalanche model in Naaim et al. [2004]). For keeping the same notation in the document, the avalanche model is hereafter denoted by $f$.

\subsection{The inputs}

The avalanche model depends on six inputs: the friction parameters $\mu$ and $\xi$, the length $l_{\text {start }}$ of the avalanche release zone, the snow depth $h_{\text {start }}$ within the release zone, the release position of the avalanche denoted by $\mathrm{x}_{\text {start }}$ and the discretized topography of the flow path, denoted by $D=(\mathbf{x}, \mathbf{z}) \in \mathbb{R}^{T \times 2}$ where $\mathbf{x} \in \mathbb{R}^{T}$ is the vector of projected runout length from the starting point of the avalanche and $\mathbf{z}=l(\mathbf{x}) \in \mathbb{R}^{T}$ is the elevation vector. $T$ is the number of points of the discretized path.

Additionally to these parameters, we included the term $\sigma$ to code the error of the digital elevation model $\mathbf{z}$ on the path's topography. To do so, a Gaussian error term is added to each element of $\mathbf{z}$ to construct a new topography $D^{\prime}=\left(\mathbf{x}, \mathbf{z}^{\prime}\right)$ for each new model run, whose elevation is calculated as follows: 


\begin{tabular}{lll}
\hline Input & Description & Distribution \\
\hline$\mu$ & Static friction coefficient & $\mathcal{U}[0.177,0.498]$ \\
$\xi$ & Turbulent friction $\left[{\left.\mathrm{m} . \mathrm{s}^{-2}\right]}^{\mathrm{h}}\right.$ & $\mathcal{U}[306.97,1475.67]$ \\
$\mathrm{h}_{\text {start }}$ & Snow depth within the release zone $[\mathrm{m}]$ & $\mathcal{U}[0.17,0.33]$ \\
$\mathrm{l}_{\text {start }}$ & Length of the release zone $[\mathrm{m}]$ & $\mathcal{U}[24,34]$ \\
$\sigma$ & Digital Elevation Model error $[\mathrm{m}]$ & $\mathcal{U}[0,0.15]$ \\
\hline
\end{tabular}

Table 2: Avalanche model: Input description and uncertainty intervals. $\mathcal{U}[a, b]$ denotes an uniform distribution in the interval $[a, b]$.

$$
\mathbf{z}^{\prime}=\mathbf{z}+\mathcal{N}\left(0, \mathbb{I}_{T} \sigma^{2}\right)
$$

where $\mathbb{I}_{T}$ is the $T \times T$ identity matrix. This error codes the imprecision of the digital elevation model which allows analysing how it affects the simulation results.

In this study, we analyzed precisely the sensitivity of the model for the simulations of a single avalanche event. The input uncertainty intervals are those corresponding to an avalanche released at the Lautaret full-scale testsite on 13 February 2013. More details about this avalanche event can be found in Pulfer et al. 2013, Heredia et al., 2020. Thus parameter (x,z) is not uncertain anymore.

For this avalanche event, the release position of the avalanche $\mathrm{x}_{\text {start }}$ is precisely known because it was fixed by the experimental team (artificial avalanche release). Thanks to the sophisticated equipment of the test-site, the uncertainty of this input can therefore be neglected. The $1_{\text {start }}$ and $\mathrm{h}_{\text {start }}$ input uncertainty intervals could be determined thanks to measures taken during the experiment. By contrast, the inputs $\mu$ and $\xi$ cannot be measured. In another study, we developed a Bayesian calibration approach to infer these inputs from the measurements made along the flow. Thus, the uncertainty intervals of $\mu$ and $\xi$ considered here correspond to the $95 \%$ credibility intervals of the posterior distribution we obtained Heredia et al. 2020. However, to avoid privileging some values in the sensitivity analysis, uniform distributions were chosen to model the $d=5$ inputs, including the friction parameters (i.e. we did not directly use their posterior distribution). The uncertain inputs of the model and their uncertainty intervals are summarized in Table 2 . 


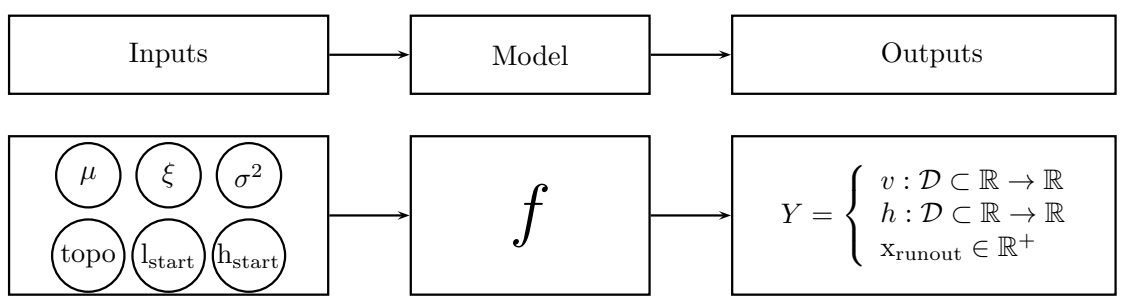

Figure 6: Avalanche model: Inputs and outputs. $\mathrm{x}_{\text {start }}$ is fixed. All other quantities are taken as random and considerd in the sensitivity analysis.

\subsection{The outputs}

The outputs of the avalanche model are the functional maximal velocity $\vec{v}$ and the functional maximal flow depth $\vec{h}$ of the avalanche on the discretized grid corresponding to the topography $D$, and the runout distance of the avalanche denoted by $\mathrm{x}_{\text {runout}}$. In other words, the model has two vectorial and one scalar outputs.

The vectors whose components are the evaluations on the discretized topography $D$ of the velocity and flow depth functional outputs are denoted $\mathbf{v}=\left(v_{1}, \ldots, v_{T}\right)$ and $\mathbf{h}=\left(h_{1}, \ldots, h_{T}\right)$, respectively. Note that we have $p=2 \times T+1$ outputs. A brief summary of our framework is drawn in Figure 6 .

The global sensitivity analysis is conducted only on a section of the path where all the avalanche simulations are flowing. More precisely, considering e.g., the velocity, our sensitivity analysis is performed on a subset of length $n, \mathbf{v}=\left\{v_{1}^{\ell}, \ldots, v_{T_{1}}^{\ell}\right\}_{\ell \in\{1, \ldots n\}}$, with $T_{1} \leq T$ and such that $v_{k}^{\ell}>0$ for all $k \in\left\{1, \ldots, T_{1}\right\}$.

Figure 7 shows the functional high density region (HDR) boxplots of 300 velocity (a) and snow depth curves (b) and the boxplot for the runout distance (c). The functional HDR plots are a tool for visualizing large amounts of functional data based in the estimation of the bivariate kernel density function of the two first components of the decomposition of the functional data [see Hyndman and Shang, 2010, for more details]. The HDR boxplots show the 50\% HDR and the 100\% HDR in light and dark gray, respectively. The modal curve is plotted with a solid line. These Figures were obtained using the $\mathrm{R}$ package rainbow developed by Hyndman and Shang 2010. 
a)

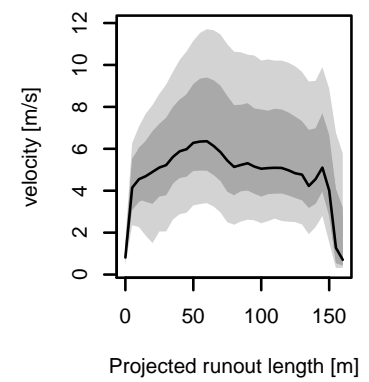

b)

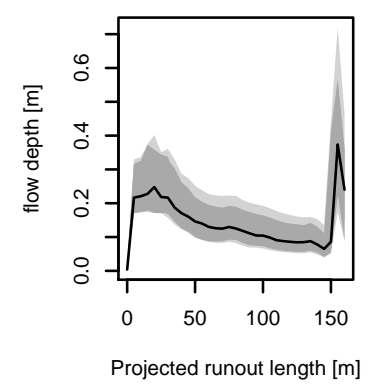

c)

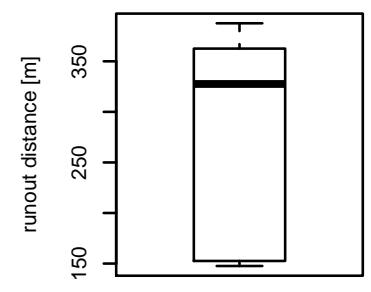

Figure 7: Avalanche model: a) functional high density region boxplots for the velocity b) functional high density region boxplots for the flow depth and, c) boxplot for the runout distance.

\subsection{Sobol' indices}

To estimate the sensitivity indices, we developed $n=300$ model simulations using the random input distributions shown in Table 2. Figure 8 shows the topography of the avalanche path and the gray box shows the region where all the avalanche simulations are flowing. The output values in this subset are used in the sensitivity analysis. Also, in Figure 8, we show the percentage of avalanches flowing in the path as function of the position along the topography.

\subsection{Scalar Sobol' indices}

The scalar Sobol' indices were calculated using $n=300$ samples and they are shown in Figure 9. For the velocity output, in the first $50 \mathrm{~m}$ of the path, the input $\mu$ is the most important, then it is followed by $\xi$. For the rest of the path, the input $\xi$ is the most important. For the flow depth output, the $h_{\text {start }}$ input is the most important. The input $\sigma^{2}$ is the less important parameter for both outputs. The scalar sensitivity indices for the runout distance are shown in Figure 10. For this output, the $h_{\text {start }}$ input is the most important. These three figures give us valuable information about the model. However, it could also be interesting to get a summarized information by computing aggregated sensitivity indices and this is done in the following section. 


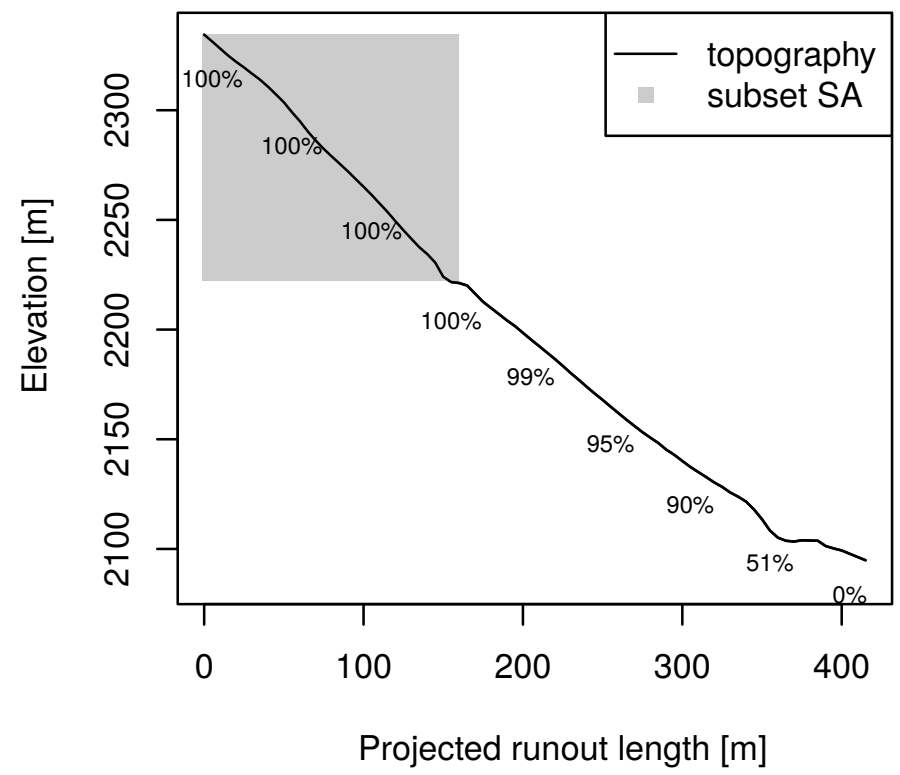

Figure 8: Avalanche model: path's topography (black line) with $T=84$ discretization points and the subset of the path in gray box $\left(T_{1}=33\right.$ discretization points) where the sensitivity analysis is performed. The panel highlights the percentage of avalanche flowing as function of the position within the path. 

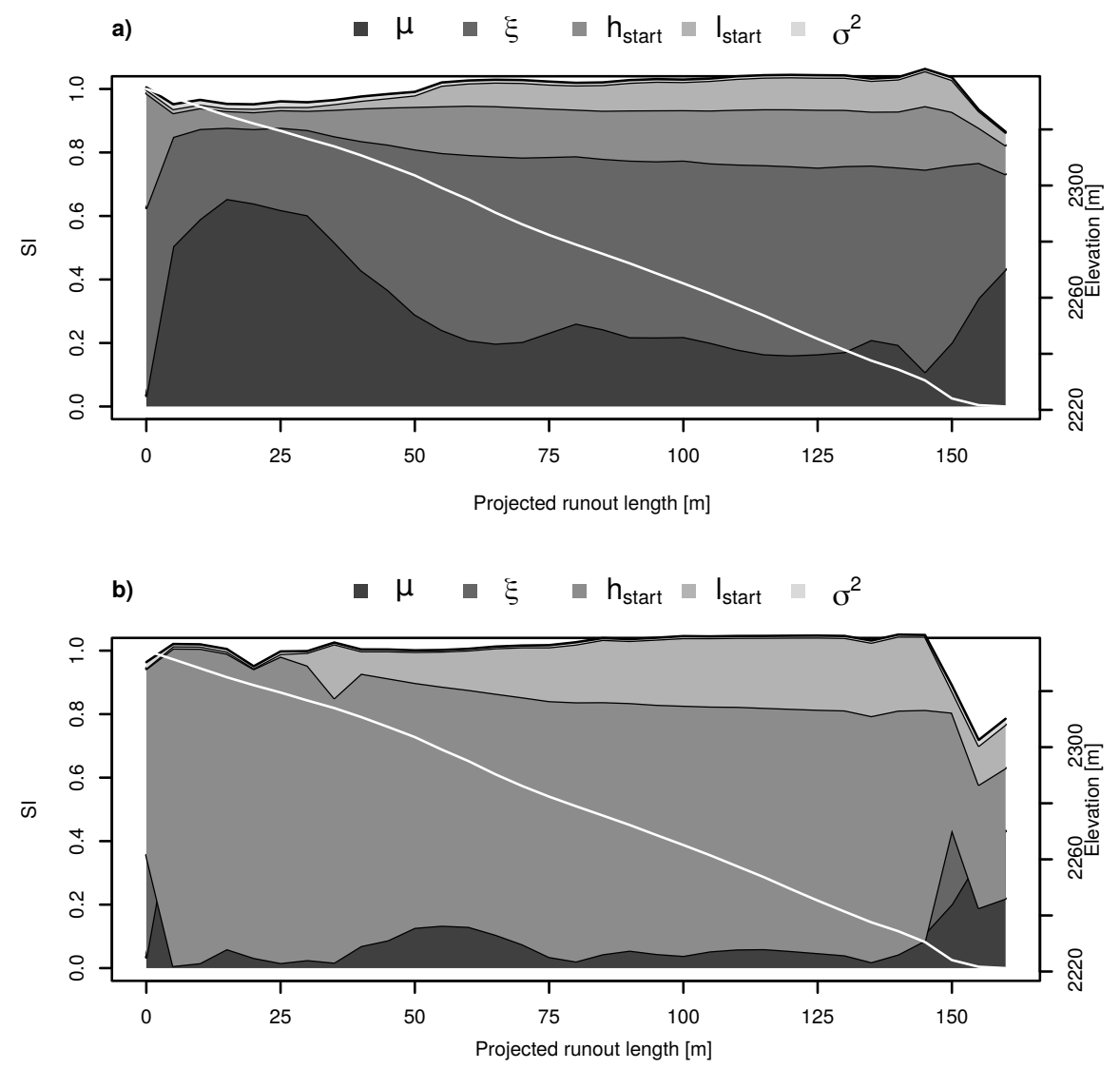

Figure 9: Avalanche model: a) scalar Sobol' indices for the velocity and b) flow depth outputs. The white line corresponds to the path's topography. 


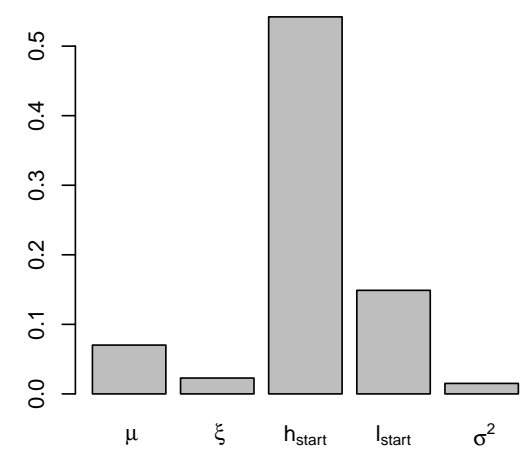

Figure 10: Avalanche model: scalar Sobol' indices for the runout distance output.

\subsection{Aggregated Sobol' indices}

To estimate the aggregated indices, we used two ways of aggregating the output information. In the first one, we performed two PCAs separately: one for the velocity output and the other one for the flow depth output. And in the second one, we used simultaneous PCA to estimate the indices of the three outputs. The results of the aggregated indices using two PCAs are shown in the panel a) of Figure 11 and using simultaneous PCA in the panel b) of Figure 11. This summarizes the importance of the inputs.

Let us analyze the results of the aggregated indices calculated when performing a PCA on each functional output: for the velocity output, the $\xi$ and $\mu$ inputs are the most relevant and for the flow depth output, the $h_{\text {start }}$ input is by far the most important. These results are consistent with findings of other studies [e.g., Barbolini and Savi, 2001, Borstad and McClung, 2009, Fischer et al., 2015.

Finally, let us analyze the results of the aggregated indices calculated when performing a simultaneous PCA for the whole set of outputs(he two functional ones and the scalar runout distance): the most important inputs are $\xi, \mathrm{h}_{\text {start }}$ and $\mu$. 
a)

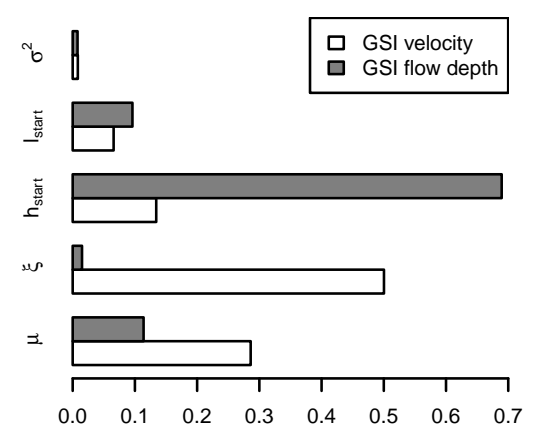

b)

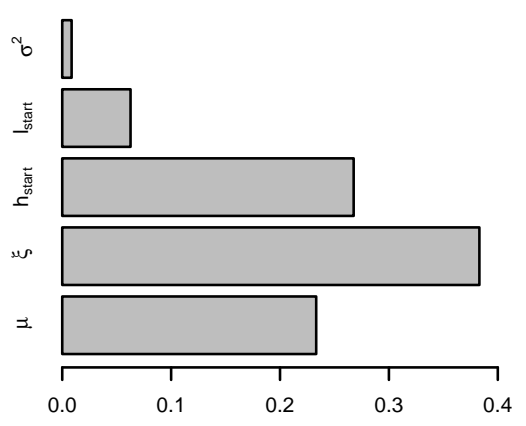

Figure 11: Avalanche model: aggregated indices estimated with two ways of aggregating the information. a) Two PCAs performed separately for the two functional outputs: one for the velocity output and the other one for the flow depth output. b) Simultaneous PCA to estimate the indices of the three outputs (the two functional ones and the scalar runout distance) altogether.

\section{Conclusions and perspectives}

In this work, we proposed a nonparametric method to estimate the aggregated Sobol' indices from a given random sample with small size, we called this method cnp. The method is based on the Nadaraya-Watson kernel smoother. Due to the small size of the sample at hand, the kernel estimation is biased. Therefore, to remove the bias of the estimation, we proposed a bias correction using bootstrapping samples based on the works of Racine 2001 and Solís 2019] before bandwidth selection based on cross-validation.

We tested the accuracy of the method on a scalar and two multivariate test cases. In general, the method $\mathrm{cnp}$ is accurate, even if the sample size is low $(n=300)$. Also, by comparing $\mathrm{cnp}$ with its no bias correction $\mathrm{np}$ variant, the results have shown that the bias is successfully removed. Nevertheless, it still exhibits small bias for very small Sobol' indices. However, the objective of our sensitivity analysis was factor prioritization and this aim is well achieved with our method.

The method was developed to estimate the aggregated indices for an avalanche model which has three outputs: the functional velocity and flow depth discretized on a given topography and the runout distance of the 
avalanche. In this work, we developed a sensitivity analysis of a single avalanche event. The event corresponds to an avalanche released at the Lautaret test-site the 13 February 2013. The results have shown that for this particular avalanche event: for the velocity output, the $\mu$ and $\xi$ inputs are the most relevant and for the snow depth output, $\mathrm{h}_{\text {start }}$ is the most important. Finally, for the runout distance output, $\mathrm{h}_{\text {start }}$ is the most important. We also showed how this information can be aggregated in one single set of indices summing up the model sensitivity which could be a very useful information for avalanche practitioners.

Note that in this work we proposed a method to quantify the importance of the inputs of a particular avalanche model but this method can be widely applied to other avalanche models. Moreover, the method could be applied to other avalanche events with the same model to generalize the results. Eventually, it could be adapted to various problems for which complex models with outputs which are both functional and scalar are employed.

In this paper, we did not provide confidence intervals (CI) associated to the estimation of Sobol' or aggregated Sobol' indices. This would be an interesting task for a future work. Bootstrap based CI are costly in that framework as they would involve two boostrap stages: one for the bias correction and one for the computation of CI.

\section{Acknowledgment}

M.B. Heredia holds a PhD grant from Labex OSUG@2020. Within the CDPTrajectories framework, this work is supported by the French National Research Agency in the framework of the "Investissements d'avenir" program (ANR-15-IDEX-02). The computations were performed using the Froggy platform of the CIMENT infrastructure (https://ciment.ujf-grenoble.fr), which is supported by the Rhône-Alpes region (GRANT CPER07 13 CIRA), the OSUG@2020 labex (reference ANR10 LABX56) and the Equip@Meso project (reference ANR-10-EQPX-29-01) of the program "Investissements d'avenir" supported by the Agence Nationale pour la Recherche. We thank Laurence Viry for her valuable advice regarding the reduction of computational cost of the cnp method and Eduardo Tusa for his advice regarding the introduction. We also thank to H. Bellot, E. Thibert and X. Ravanat for their help during the avalanche release operation and for collecting the data. 


\section{Appendix}

In this appendix, we want to compare the accuracy of Sobol' index estimation for the mass-spring test case when using a single sample of size $n=200$ for both PCA and Sobol' index estimation or two independent samples of size $n=100$, one for PCA, the other one for Sobol' index estimation. Boxplots presented in Figure 12 were obtained with $N=100$ independent replications.

\section{References}

C. Ancey and M. Meunier. Estimating bulk rheological properties of flowing snow avalanches from field data. Journal of Geophysical Research: Earth Surface, 109(F1), 2004. doi: 10.1029/2003JF000036. URL https:// agupubs.onlinelibrary.wiley.com/doi/abs/10.1029/2003JF000036.

Christophe Ancey. Dynamique des avalanches. Presses Polytechniques et Universitaires romandes \& Cemagref, 2006.

Anestis Antoniadis, Cline Helbert, Clmentine Prieur, and Laurence Viry. Spatio-temporal metamodeling for West African monsoon. Environmetrics, 23(1):24-36, 2012. doi: 10.1002/env.1134. URL https:// onlinelibrary.wiley.com/doi/abs/10.1002/env.1134.

Isadora Antoniano-Villalobos, Emanuele Borgonovo, and Xuefei Lu. Bayesian estimation of probabilistic sensitivity measures. arXiv e-prints, art. arXiv:1907.09424, Jul 2019.

Benjamin Auder and B. Iooss. Global sensitivity analysis based on entropy. In Safety, Reliability and Risk Analysis - Proceedings of the ESREL 2008 Conference, pages 2107-2115, Valencia, Spain, 2008. CRC Press.

M. Barbolini and F. Savi. Estimate of uncertainties in avalanche hazard mapping. Annals of Glaciology, 32:299-305, 2001. doi: 10.3189/ 172756401781819373.

Emanuele Borgonovo and Elmar Plischke. Sensitivity analysis: A review of recent advances. European Journal of Operational Research, 248(3): 869-887, 2016. doi: 10.1016/j.ejor.2015.06.032. URL https://doi.org/ $10.1016 /$ j.ejor.2015.06.032. 

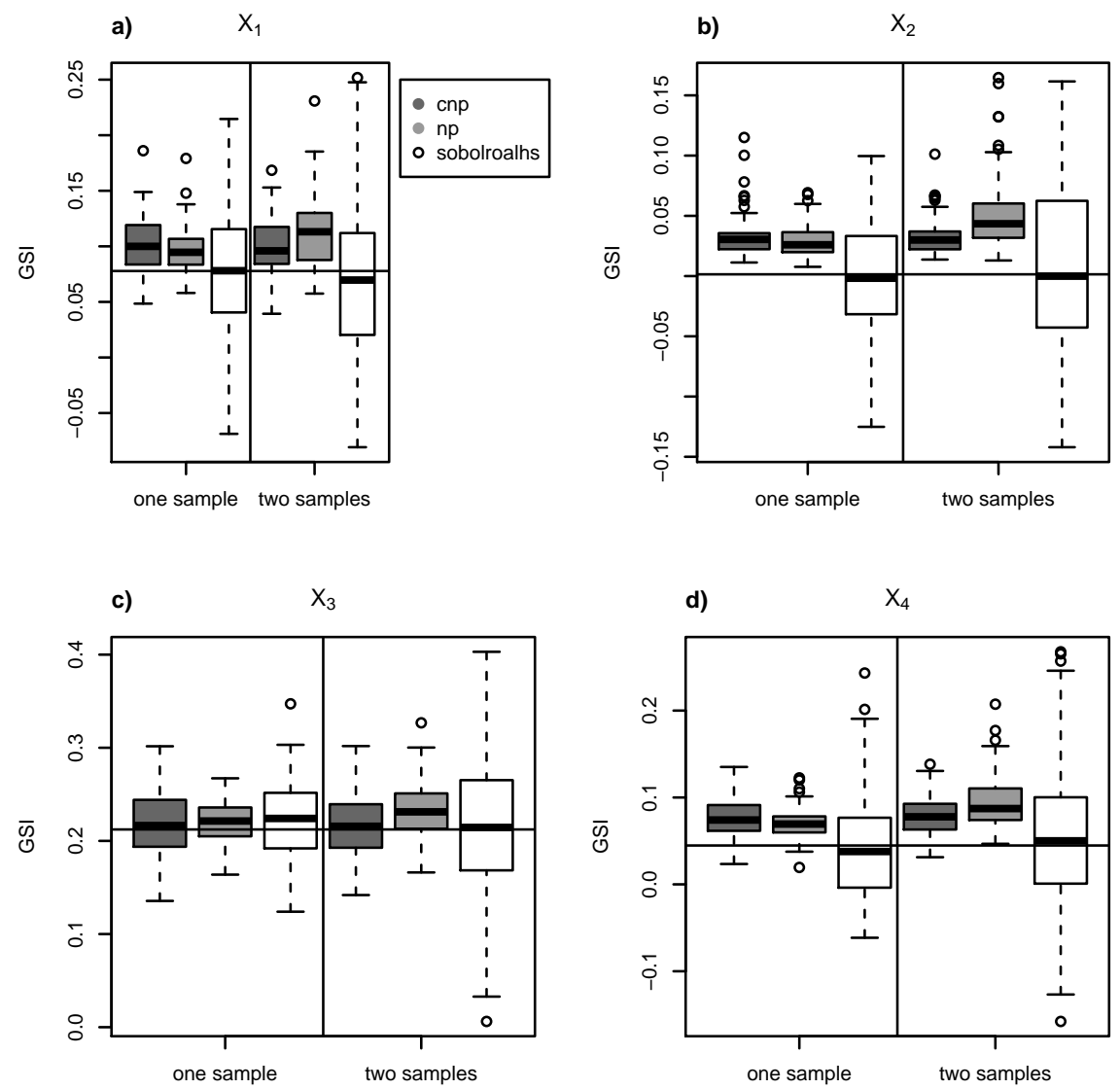

Figure 12: Mass-spring model: Estimation of the aggregated Sobol' indices using cnp, $\mathrm{np}$ and sobolroalhs. Boxplots were drawn from $\mathrm{N}=100$ samples of sizes 200. The estimation results using the same sample to estimate the indices and the PCs (one sample) and to estimate the indices and the PCs using two samples are shown. To use cnp, we set $B=100$. 
Emanuele Borgonovo, Gordon B. Hazen, and Elmar Plischke. A Common Rationale for Global Sensitivity Measures and Their Estimation. Risk Analysis, 36(10):1871-1895, 2016. doi: 10.1111/risa.12555. URL https: //onlinelibrary.wiley.com/doi/abs/10.1111/risa.12555.

Christopher P. Borstad and D.M. McClung. Sensitivity analyses in snow avalanche dynamics modeling and implications when modeling extreme events. Canadian Geotechnical Journal, 46(9):1024-1033, 2009. doi: 10. 1139/T09-042. URL https://doi.org/10.1139/T09-042.

Baptiste Broto, François Bachoc, and Marine Depecker. Variance reduction for estimation of Shapley effects and adaptation to unknown input distribution. arXiv e-prints, art. arXiv:1812.09168, December 2018.

R. Bühler, C. Argue, B. Jamieson, and A. Jones. Sensitivity Analysis of the RAMMS Avalanche Dynamics Model in a Canadian Transitional Snow Climate. International Snow Science Workshop Proceedings 2018, Innsbruck, Austria, 2018.

Yves Bühler, Marc Christen, Julia Kowalski, and Perry Bartelt. Sensitivity of snow avalanche simulations to digital elevation model quality and resolution. Annals of Glaciology, 52(58):72-80, 2011. doi: 10.3189/172756411797252121.

Katherine Campbell, Michael D. McKay, and Brian J. Williams. Sensitivity analysis when model outputs are functions. Reliability Engineering \& System Safety, 91(10):1468-1472, 2006. ISSN 0951-8320. doi: https://doi. org/10.1016/j.ress.2005.11.049. URL http://www.sciencedirect.com/ science/article/pii/S0951832005002565. The Fourth International Conference on Sensitivity Analysis of Model Output (SAMO 2004).

Sebastien Da Veiga, Francois Wahl, and Fabrice Gamboa. Local polynomial estimation for sensitivity analysis on models with correlated inputs. Technometrics, 51(4):452-463, 2009. doi: 10.1198/TECH.2009.08124. URL https://doi.org/10.1198/TECH.2009.08124.

N. Eckert, E. Parent, M. Naaim, and D. Richard. Bayesian stochastic modelling for avalanche predetermination: from a general system framework to return period computations. Stochastic Environmental Research and Risk Assessment, pages 185-206, Feb 2008. ISSN 14363259. doi: 10.1007/s00477-007-0107-4. URL https://doi.org/10.1007/ s00477-007-0107-4. 
N. Eckert, M. Naaim, and E. Parent. Long-term avalanche hazard assessment with a Bayesian depth averaged propagation model. Journal of Glaciology, 56:563-586, October 2010. doi: 10.3189/002214310793146331.

Nicolas Eckert, Mohamed Naaim, Florie Giacona, Philomène Favier, Aurore Lavigne, Didier Richard, Franck Bourrier, and Eric Parent. Repenser les fondements du zonage réglementaire des risques en montagne récurrents. La Houille Blanche, (2):38-67, 2018.

B. Efron and C. Stein. The jackknife estimate of variance. Ann. Statist., 9 (3):586-596, 05 1981. doi: 10.1214/aos/1176345462. URL https://doi. org/10.1214/aos/1176345462.

P Favier, N Eckert, David Bertrand, and M Naaim. Sensitivity of avalanche risk to vulnerability relations. Cold regions science and technology, 108: 163-177, 2014.

J.-T. Fischer. A novel approach to evaluate and compare computational snow avalanche simulation. Natural Hazards and Earth System Sciences, 13(6):1655-1667, 2013. doi: 10.5194/nhess-13-1655-2013. URL https: //www.nat-hazards-earth-syst-sci.net/13/1655/2013/.

Jan-Thomas Fischer, Andreas Kofler, Wolfgang Fellin, Matthias Granig, and Karl Kleemayr. Multivariate parameter optimization for computational snow avalanche simulation. Journal of Glaciology, 61(229):875-888, 2015. doi: $10.3189 / 2015 J o G 14 J 168$.

F. Gamboa, A. Janon, T. Klein, and A. Lagnoux. Sensitivity analysis for multidimensional and functional outputs. ArXiv e-prints, November 2013.

Fabrice Gamboa, Alexandre Janon, Thierry Klein, and Agnès Lagnoux. Sensitivity indices for multivariate outputs. Comptes Rendus Mathematique, 351(7):307 - 310, 2013. ISSN 1631-073X. doi: https://doi. org/10.1016/j.crma.2013.04.016. URL http://www.sciencedirect.com/ science/article/pii/S1631073X13000824

Urs Gruber and Stefan Margreth. Winter 1999: a valuable test of the avalanche-hazard mapping procedure in Switzerland. Annals of Glaciology, 32:328332, 2001. doi: 10.3189/172756401781819238.

M. B. Heredia, Nicolas Eckert, Clémentine Prieur, and Thibert Emmanuel. Bayesian calibration of an avalanche model from autocorrelated measurements along the flow: application to velocities extracted from photogrammetric images. Journal of Glaciology, 2020. Forthcoming. 
Wassily Hoeffding. A Class of Statistics with Asymptotically Normal Distribution. The Annals of Mathematical Statistics, 19(3):293-325, 1948. ISSN 00034851. URL http://www.jstor.org/stable/2235637.

Rob J. Hyndman and Han Lin Shang. Rainbow plots, bagplots, and boxplots for functional data. Journal of Computational and Graphical Statistics, 19 (1):29-45, 2010. doi: 10.1198/jcgs.2009.08158. URL https://doi.org/ $10.1198 / \mathrm{jcgs} .2009 .08158$.

B. Iooss and C. Prieur. Shapley effects for sensitivity analysis with correlated inputs: comparisons with Sobol' indices, numerical estimation and applications. ArXiv e-prints, July 2017.

Bertrand Iooss and Paul Lemaître. A Review on Global Sensitivity Analysis Methods, pages 101-122. Springer US, Boston, MA, 2015. ISBN 978-14899-7547-8. doi: 10.1007/978-1-4899-7547-8_5. URL https://doi.org/ 10.1007/978-1-4899-7547-8_5.

Bruce Jamieson, Stefan Margreth, and Alan Jones. Application and limitations of dynamic models for snow avalanche hazard mapping. Proceedings of the ISSW 2008. Wistler, Canada, pages 730-739, 012008.

Matieyendou Lamboni, David Makowski, Simon Lehuger, Benoit Gabrielle, and Herv Monod. Multivariate global sensitivity analysis for dynamic crop models. Field Crops Research, 113(3):312 - 320, 2009. ISSN 03784290. doi: https://doi.org/10.1016/j.fcr.2009.06.007. URL http://www. sciencedirect.com/science/article/pii/S0378429009001531.

N. Locantore, J. S. Marron, D. G. Simpson, N. Tripoli, J. T. Zhang, K. L. Cohen, Graciela Boente, Ricardo Fraiman, Babette Brumback, Christophe Croux, Jianqing Fan, Alois Kneip, John I. Marden, Daniel Peña, Javier Prieto, Jim O. Ramsay, Mariano J. Valderrama, Ana M. Aguilera, N. Locantore, J. S. Marron, D. G. Simpson, N. Tripoli, J. T. Zhang, and K. L. Cohen. Robust principal component analysis for functional data. Test, 8(1):1-73, Jun 1999. ISSN 1863-8260. doi: 10.1007/BF02595862. URL https://doi.org/10.1007/BF02595862.

Michel Loève. Probability Theory. Springer, New York, 3rd edition, 1963.

David McClung and Peter A Schaerer. The avalanche handbook. The Mountaineers Books, 2006. 
Mohamed Naaim, Florence Naaim-Bouvet, Thierry Faug, and Alexi Bouchet. Dense snow avalanche modeling: flow, erosion, deposition and obstacle effects. Cold Regions Science and Technology, 39(2):193 - 204, 2004. ISSN 0165-232X. doi: https://doi.org/10.1016/j.coldregions.2004. 07.001. URL http://www.sciencedirect.com/science/article/pii/ S0165232X04000643. Snow And Avalanches: Papers Presented At The European Geophysical Union Conference, Nice, April 2003. Dedicated To The Avalanche Dynamics Pioneer Dr. B. Salm.

Mohamed Naaim, Thierry Faug, Florence Naaim, and Nicolas Eckert. Return period calculation and passive structure design at the taconnaz avalanche path, france. Annals of Glaciology, 51(54):89-97, 2010.

Mohamed Naaim, Yves Durand, Nicolas Eckert, and Guillaume Chambon. Dense avalanche friction coefficients: influence of physical properties of snow. Journal of Glaciology, 59(216):771-782, 2013. doi: 10.3189/2013JoG12J205.

E. A. Nadaraya. On estimating regression. Theory of Probability \& Its Applications, 9(1):141-142, 1964. doi: 10.1137/1109020. URL https: //doi.org/10.1137/1109020.

Simon Nanty, Céline Helbert, Amandine Marrel, Nadia Pérot, and Clémentine Prieur. Uncertainty quantification for functional dependent random variables. Computational Statistics, 32(2):559-583, Jun 2017. ISSN 1613-9658. doi: 10.1007/s00180-016-0676-0. URL https: //doi.org/10.1007/s00180-016-0676-0.

A. Owen. Sobol' Indices and Shapley Value. SIAM/ASA Journal on Uncertainty Quantification, 2(1):245-251, 2014. doi: 10.1137/130936233. URL https://doi.org/10.1137/130936233.

Karl Pearson. LIII. On lines and planes of closest fit to systems of points in space. The London, Edinburgh, and Dublin Philosophical Magazine and Journal of Science, 2(11):559-572, 1901.

Elmar Plischke. An effective algorithm for computing global sensitivity indices (EASI). Reliability Engineering \& System Safety, 95(4):354 360, 2010. ISSN 0951-8320. doi: https://doi.org/10.1016/j.ress.2009. 11.005. URL http://www.sciencedirect.com/science/article/pii/ S0951832009002579. 
Elmar Plischke, Emanuele Borgonovo, and Curtis L. Smith. Global sensitivity measures from given data. European Journal of Operational Research, 226(3):536-550, 2013. doi: 10.1016/j.ejor.2012.11.06. URL https: //ideas.repec.org/a/eee/ejores/v226y2013i3p536-550.html.

G. Pulfer, M. Naaim, E. Thibert, and A. Soruco. Retrieving avalanche basal friction law from high rate positioning of avalanches. In International Snow Science Workshop (ISSW), Grenoble - Chamonix MontBlanc, France, October 2013. Irstea, ANENA, Meteo France. URL https://hal.archives-ouvertes.fr/hal-00951328.

R Core Team. R: A Language and Environment for Statistical Computing. R Foundation for Statistical Computing, Vienna, Austria, 2019. URL https://www.R-project.org/

Jeff Racine. Bias-Corrected Kernel Regression. Journal of Quantitative Economics, 17(1):25-42, 2001.

J. O. Ramsay and B. W. Silverman. Functional Data Analysis. Springer Series in Statistics. Springer, New York, 2nd edition, June 2005. ISBN 038740080X. URL http://www.worldcat.org/isbn/038740080X.

A. Saltelli, S. Tarantola, and K. P.-S. Chan. A quantitative modelindependent method for global sensitivity analysis of model output. Technometrics, 41(1):39-56, 1999. doi: 10.1080/00401706.1999. 10485594. URL https://www.tandfonline.com/doi/abs/10.1080/ 00401706.1999 .10485594 .

A. Saltelli, K. Chan, and E.M. Scott. Sensitivity Analysis: Gauging the Worth of Scientific Models. Wiley Series in Probability and Statistics. Wiley, New York, United States, 2000. ISBN 9780471998921.

Andrea Saltelli, Stefano Tarantola, Francesca Campolongo, and Marco Ratto. Sensitivity Analysis in Practice: A Guide to Assessing Scientific Models. Halsted Press, New York, NY, USA, 2004. ISBN 0470870931.

Andrea Saltelli, Marco Ratto, Terry Andres, Francesca Campolongo, Jessica Cariboni, Debora Gatelli, Michaela Saisana, and Stefano Tarantola. Global sensitivity analysis. The primer. John Wiley, West Sussex PO19 8SQ, England, 2008. ISBN 9780470059975.

I. M. Sobol'. Sensitivity analysis for non-linear mathematical models. Mathematical Modelling and Computational Experiment, 1(4):407-414, 1993. 
Maikol Solís. Non-parametric estimation of the first-order sobol indices with bootstrap bandwidth. Communications in Statistics - Simulation and Computation, Taylor \& Francis, 0(0):1-16, 2019. doi: 10.1080/03610918. 2019.1655575 .

S. Tarantola, D. Gatelli, and T.A. Mara. Random balance designs for the estimation of first order global sensitivity indices. Reliability Engineering E System Safety, 91(6):717 - 727, 2006. ISSN 0951-8320. doi: https: //doi.org/10.1016/j.ress.2005.06.003. URL http://www.sciencedirect. com/science/article/pii/S0951832005001444.

Emmanuel Thibert, Herv Bellot, Xavier Ravanat, Frdric Ousset, Gatan Pulfer, Mohamed Naaim, Pascal Hagenmuller, Florence Naaim-Bouvet, Thierry Faug, Koichi Nishimura, Yoichi Ito, Djebar Baroudi, Alexander Prokop, Peter Schon, Alvaro Soruco, Christian Vincent, Ali Limam, and Raphaele Héno. The full-scale avalanche test-site at Lautaret Pass (French Alps). Cold Regions Science and Technology, 115:30 - 41, 2015. ISSN 0165-232X. doi: https://doi.org/10.1016/j.coldregions.2015. 03.005. URL http://www.sciencedirect.com/science/article/pii/ S0165232X15000622.

Jean-Yves Tissot and Clémentine Prieur. A randomized Orthogonal Arraybased procedure for the estimation of first- and second-order Sobol' indices. Journal of Statistical Computation and Simulation, 85(7):13581381, 2015. doi: 10.1080/00949655.2014.971799. URL https://hal. archives-ouvertes.fr/hal-00743964.

Alexandre B. Tsybakov. Introduction to Nonparametric Estimation. Springer Publishing Company, Incorporated, Springer-Verlag New York, 1st edition, 2008. ISBN 0387790519, 9780387790510.

Roberto Viviani, Georg Grön, and Manfred Spitzer. Functional principal component analysis of fMRI data. Human Brain Mapping, 24(2):109-129, 2005. doi: 10.1002/hbm.20074. URL https://onlinelibrary.wiley. com/doi/abs/10.1002/hbm.20074.

Geoffrey S. Watson. Smooth regression analysis. Sankhyā: The Indian Journal of Statistics; Series A, 26:359-372, 1964.

Fang Yao, Hans-Georg Müller, and Jane-Ling Wang. Functional data analysis for sparse longitudinal data. Journal of the American Statistical Association, 100(470):577-590, 2005. doi: 10.1198/016214504000001745. URL https://doi.org/10.1198/016214504000001745. 